\title{
Extracavitary Manifestation of Primary Effusion Lymphoma as a Right Atrial Mass
}

\author{
Creticus P. Marak ${ }^{\mathrm{a}}$ Ana M. Ponea ${ }^{\mathrm{a}}$ Chang Shim $^{\mathrm{a}} \quad$ Shagufta Shaheen $^{\mathrm{b}}$ \\ Achuta K. Guddati ${ }^{b}$ \\ ${ }^{a}$ Division of Pulmonary and Critical Care Medicine, Montefiore Hospital, Albert Einstein \\ College of Medicine, Yeshiva University, New York, N.Y., and ${ }^{b}$ Department of Internal \\ Medicine, Massachusetts General Hospital, Harvard Medical School, Harvard University, \\ Boston, Mass., USA
}

\section{Key Words}

Primary effusion lymphoma $\cdot$ Right atrial mass $\cdot$ R-EPOCH $\cdot$ Human herpes virus 8

\begin{abstract}
Primary effusion lymphoma (PEL) is a subset of large B cell lymphomas and has been mostly associated with human immunodeficiency virus infection. Rare cases have been reported in organ transplant recipients and chronic hepatitis $C$ patients. It typically presents as an effusion in the pleural and pericardial spaces but rarely disseminates. However, involvement of the gastrointestinal tract, lymph nodes and bone marrow has been reported. Diagnosis is based on characteristic clinical, histopathological and immunohistochemical features. We present a case with a right atrial mass which tested positive for human herpes virus 8 (HHV8), CD20, CD30 and lambda light chains and negative for CD138, kappa light chain, PAX5, Epstein-Barr virus, latent membrane protein 1, CD2, CD3, CD8 and CD56. Bilateral pleural effusions and pericardial effusions were noted which tested positive for HHV-8, CD30 and $\mathrm{CD} 45$. The patient responded well to the R-EPOCH regimen with complete resolution of the effusions and a significant decrease in the size of the right atrial mass. This case report illustrates the atypical manifestation of PEL as a right atrial mass.
\end{abstract}

\section{Introduction}

Primary effusion lymphoma (PEL) is a subset of non-Hodgkin's lymphoma (NHL) and was first described in 1989 [1]. This entity was noted in human immunodeficiency virus 
(HIV) patients, especially after NHL in patients with HIV had been determined to be an AIDSdefining illness [2]. A malignant lymphoblastic effusion when associated with human herpes virus 8 (HHV-8) infections is defined as PEL. Most of these affected patients are also coinfected with Epstein-Barr virus (EBV). HHV-8 is endemic in sub-Saharan Africa and the Mediterranean region of the world [3]. Latency-associated nuclear antigen-1 (LANA-1) is a gene product of HHV-8, which is essential for viral DNA maintenance during replication. This protein binds to the p53 and retinoblastoma genes and inhibits tumor suppression by impairing apoptosis [4]. Detection of LANA-1 by immunohistochemistry is considered the standard assay for confirming HHV-8 infection. The post-germinal B cells are considered the cells of origin for PEL [5]. PEL cells exhibit markers characteristic of plasma cells (CD138) and lymphocyte activation (CD30, CD38, CD71 and HLA-DR) but typical B cell and T cell markers are absent (CD19, CD20, CD79a, CD3, CD4 and CD8) [6, 7]. However, no chromosomal abnormalities in terms of translocations and abnormal chromosomal numbers have been detected [8]. PEL clinically manifests as a mass effect caused by the accumulation of fluid laden with malignant cells in body cavities. In the initial presentation, it is rarely detected beyond the pleural, pericardial and peritoneal surfaces. Extracavitary presentations have been reported, principally involving the gastrointestinal tract $[9,10]$. In this case report, we present the clinical course of PEL in a patient with HIV who was found to have a right atrial mass in addition to pleural and pericardial effusions. This case is unique not only for its presentation as a right atrial mass but also for its favorable response to chemotherapy.

\section{Case Presentation}

A 35-year-old Hispanic male with AIDS without prior opportunistic infections who was recently started on antiretroviral therapy was referred from an outside hospital for the management of pericardial and bilateral pleural effusions. One month earlier, he had presented to the same hospital with intermittent fevers, progressive weakness and 20pound weight loss. Evaluation during that admission was significant for generalized lymphadenopathy and thrombocytopenia of $30,000 / \mathrm{mm}^{3}$. A computed tomography (CT) scan of the chest revealed a right atrial mass and mediastinal lymphadenopathy. His CD4 and viral load were 156 and 4 million copies, respectively. Bone marrow and left inguinal lymph node biopsies were nondiagnostic. He was discharged home on bactrim, truvada, ritonavir and darunavir. During his second hospitalization, he presented with syncope with no other localizing symptoms. On examination, he was in mild discomfort but otherwise afebrile and hemodynamically stable, with distant heart sounds and decreased air entry at the bases. A CT scan showed mediastinal adenopathy, bilateral pleural effusions, a pericardial effusion and a right atrial mass (fig. 1a). A cardiac echocardiogram showed a right atrial mass and a large pericardial effusion with tamponade physiology (fig. 1c). Thoracentesis of the right pleural effusion showed a lymphocytic predominant exudative effusion. Therapeutic pericardiocentesis with removal of $560 \mathrm{ml}$ bloody fluid was performed. Fluid cytology showed large lymphoblastic cells with a positive immunohistochemistry staining for HHV-8, CD30 and CD45. Staining for EBV, B and T cell markers was negative. The biopsy of the right atrial mass via transvenous route showed large lymphoblastic cells with positive staining for HHV-8, CD20, CD30 and lambda light chains (fig. 2a, b). Staining for CD138, kappa light chain, PAX5, EBV, latent membrane protein 1 (LMP1), CD2, CD3, CD8 and CD56 was negative. A diagnosis of PEL with extracavitary involvement was made based on the presence of lymphoblastic cells and immunohistochemical staining. The patient was 
continued on antiretroviral therapy, started on the R-EPOCH regimen as an inpatient and discharged home with outpatient follow-up appointments to oncology and infectious disease clinics. Subsequent imaging showed resolution of the pleural effusions and a significant decrease in the size of the right atrial mass (fig. 1b, d).

\section{Discussion}

PEL is a rare entity mostly described in patients infected with HIV. It accounts for 1-4\% of AIDS-related lymphomas [7]. Effective treatment with highly active retroviral treatment has contributed to the decrease in the incidence of NHL. Very rarely, PEL has been reported in patients with organ transplant recipients and chronic hepatitis C virus infection [11-13]. It is a subtype of diffuse large B cell lymphoma with a unique constellation of clinical, morphologic, immunophenotypic and molecular characteristics. There is no clear association with the CD4 count and viral load in HIV-infected patients [14, 15]. HHV-8 has been implicated in the pathogenesis of PEL [16, 17]. It predominantly affects males [14] and is characterized by a symptomatic serous effusion with involvement of the pleura (60-90\%) and pericardium (30\%) causing dyspnea, the peritoneum (30-60\%) causing abdominal discomfort and distension, joint spaces causing joint swelling and, rarely, the meninges [14]. Chest roentgenograms typically show pleural and pericardial thickening with an effusion in the absence of parenchymal abnormalities, masses or mediastinal enlargement $[18,19]$. It rarely disseminates [14] but can present as solid tumors involving the gastrointestinal tract, lymph nodes and bone marrow. Due to its unique liquid phase pattern of growth, fluid cytology is usually positive for malignant cells [16]. It is characterized by large multinucleated and multilobated cells with prominent nucleoli and abundant deep basophilic cytoplasm containing small clear vacuoles [11]. Immunohistochemical staining is positive for plasma cell markers (CD45, CD30, CD38, CD71, and CD138) but negative for B cell (CD19, CD20 and CD79a) and T cell (CD3, CD4 and CD8) markers. HHV-8 positivity (LANA-1) in the nuclei of malignant cells is important for clinching the diagnosis. Despite frequent coinfection with EBV, staining for LMP1 is negative [20].

Since PEL is a rare disease, treatment options are not well defined. There are no prospective trials and only a few retrospective treatment series are currently known. The median survival without treatment is 2-3 months [21] and increases to about 6 months with treatment [22]. Different chemotherapy regimens have been used to treat this condition (CHOP, hyper-CVAD and rituximab) [7, 23]. High-dose chemotherapy followed by hematopoietic stem cell transplantation has generated mixed results and these data are mostly from case reports [24, 25]. In patients infected with HIV, a combination of antiretroviral therapy with chemotherapy has superior response than either modality used alone [14, 23]. Radiation therapy can be used for palliation in patients who are not candidates for chemotherapy. Chemotherapy has been used with some success in HIV-negative patients. Cytology and biopsy of the pericardial effusion and right atrial mass in our patient were positive for PEL. He had significant mediastinal lymphadenopathy and bilateral pleural effusions, which we thought were likely due to PEL. His mediastinal lymph nodes were not sampled and the pleural fluid was not sent for flow cytometry. Immunohistochemical staining of his right atrial mass biopsy was positive for HHV-8 and plasma cell markers; however, the cells were positive for B (CD20) and T (CD4) cell markers as well. To our knowledge, there has been only one case report of PEL presenting as intracardiac mass, and the patient responded favorably to chemotherapy [26]. This is probably one of the few cases of PEL with both cavitary and extracavitary involvements. Findings of an intracardiac mass 
in conjunction with pericardial and probably pleural and mediastinal lymph node involvement, cell markers positive for plasma cells, and B and T cells altogether make our patient a unique case of PEL occurring in HIV infection.

\section{Disclosure Statement}

The authors declare that there are no conflicts of interest regarding this paper.

\section{References}

1 Knowles DM, Inghirami G, Ubriaco A, Dalla-Favera R: Molecular genetic analysis of three AIDS-associated neoplasms of uncertain lineage demonstrates their B-cell derivation and the possible pathogenetic role of the Epstein-Barr virus. Blood 1989;73:792-799.

$\checkmark 2$ Centers for Disease Control (CDC): Revision of the case definition of acquired immunodeficiency syndrome for national reporting - United States. MMWR Morb Mortal Wkly Rep 1985;34:373-375.

-3 Dukers NH, Rezza G: Human herpesvirus 8 epidemiology: what we do and do not know. AIDS 2003;17:1717-1730.

-4 Friborg J Jr, Kong W, Hottiger MO, Nabel GJ: p53 inhibition by the LANA protein of KSHV protects against cell death. Nature 1999;402:889-894.

5 Matolcsy A, Nador RG, Cesarman E, Knowles DM: Immunoglobulin VH gene mutational analysis suggests that primary effusion lymphomas derive from different stages of B cell maturation. Am J Pathol 1998;153:1609-1614.

6 Gaidano G, Gloghini A, Gattei V, et al: Association of Kaposi's sarcoma-associated herpesvirus-positive primary effusion lymphoma with expression of the CD138/syndecan-1 antigen. Blood 1997;90:4894-4900.

7 Chen YB, Rahemtullah A, Hochberg E: Primary effusion lymphoma. Oncologist 2007;12:569-576.

-8 Boulanger E, Agbalika F, Maarek 0, et al: A clinical, molecular and cytogenetic study of 12 cases of human herpesvirus 8 associated primary effusion lymphoma in HIV-infected patients. Hematol J 2001;2:172-179.

$>9$ Costes V, Faumont N, Cesarman E, et al: Human herpesvirus-8-associated lymphoma of the bowel in human immunodeficiency virus-positive patients without history of primary effusion lymphoma. Hum Pathol 2002;33:846-849.

10 Carbone A, Gloghini A, Vaccher E, et al: Kaposi's sarcoma-associated herpesvirus/human herpesvirus type 8-positive solid lymphomas: a tissue-based variant of primary effusion lymphoma. J Mol Diagn 2005;7:1727.

11 Jones D, Ballestas ME, Kaye KM, et al: Primary-effusion lymphoma and Kaposi's sarcoma in a cardiactransplant recipient. N Engl J Med 1998;339:444-449.

12 Melo NC, Sales MM, Santana AN, Costalonga EC, Pedreira AB, Ianhez LE: Pleural primary effusion lymphoma in a renal transplant recipient. Am J Transplant 2008;8:906-907.

13 Nakayama-Ichiyama S, Yokote T, Kobayashi K, et al: Primary effusion lymphoma of T-cell origin with $\mathrm{t}(7 ; 8)(\mathrm{q} 32 ; \mathrm{q} 13)$ in an HIV-negative patient with HCV-related liver cirrhosis and hepatocellular carcinoma positive for HHV6 and HHV8. Ann Hematol 2011;90:1229-1231.

14 Simonelli C, Spina M, Cinelli R, et al: Clinical features and outcome of primary effusion lymphoma in HIVinfected patients: a single-institution study. J Clin Oncol 2003;21:3948-3954.

15 Mbulaiteye SM, Biggar RJ, Goedert JJ, Engels EA: Pleural and peritoneal lymphoma among people with AIDS in the United States. J Acquir Immune Defic Syndr 2002;29:418-421.

16 Nador RG, Cesarman E, Chadburn A, et al: Primary effusion lymphoma: a distinct clinicopathologic entity associated with the Kaposi's sarcoma-associated herpes virus. Blood 1996;88:645-656.

17 Karcher DS, Alkan S: Human herpesvirus-8-associated body cavity-based lymphoma in human immunodeficiency virus-infected patients: a unique B-cell neoplasm. Hum Pathol 1997;28:801-808.

18 Cesarman E, Chang Y, Moore PS, Said JW, Knowles DM: Kaposi's sarcoma-associated herpesvirus-like DNA sequences in AIDS-related body-cavity-based lymphomas. N Engl J Med 1995;332:1186-1191.

19 Morassut S, Vaccher E, Balestreri L, et al: HIV-associated human herpesvirus 8-positive primary lymphomatous effusions: radiologic findings in six patients. Radiology 1997;205:459-463.

20 Ascoli V, Mastroianni CM, Galati V, et al: Primary effusion lymphoma containing human herpesvirus 8 DNA in two AIDS patients with Kaposi's sarcoma. Haematologica 1998;83:8-12.

-21 Komanduri KV, Luce JA, McGrath MS, Herndier BG, Ng VL: The natural history and molecular heterogeneity of HIV-associated primary malignant lymphomatous effusions. J Acquir Immune Defic Syndr Hum Retrovirol 1996;13:215-226.

22 Boulanger E, Daniel MT, Agbalika F, Oksenhendler E: Combined chemotherapy including high-dose methotrexate in KSHV/HHV8-associated primary effusion lymphoma. Am J Hematol 2003;73:143-148. 


\section{Case Reports in Oncology}

\begin{tabular}{l|l}
\hline Case Rep Oncol 2013;6:114-118 \\
\hline DOI: $\underline{10.1159 / 000346838}$ & $\begin{array}{l}\text { ○ 2013 S. Karger AG, Basel } \\
\text { www.karger.com/cro }\end{array}$ \\
\hline
\end{tabular}

Marak et al.: Extracavitary Manifestation of Primary Effusion Lymphoma as a Right Atrial Mass

23 Carbone A, Gloghini A: KSHV/HHV8-associated lymphomas. Br J Haematol 2008;140:13-24.

-24 Waddington TW, Aboulafia DM: Failure to eradicate AIDS-associated primary effusion lymphoma with highdose chemotherapy and autologous stem cell reinfusion: case report and literature review. AIDS Patient Care STDS 2004;18:67-73.

25 Won JH, Han SH, Bae SB, et al: Successful eradication of relapsed primary effusion lymphoma with high-dose chemotherapy and autologous stem cell transplantation in a patient seronegative for human immunodeficiency virus. Int J Hematol 2006;83:328-330.

26 Kriekard P, Garcia JA, Nardi-Korver L, Krantz MJ: Tumor melt: primary effusion lymphoma of the heart. Am J Med 2012;125:e5-e6.
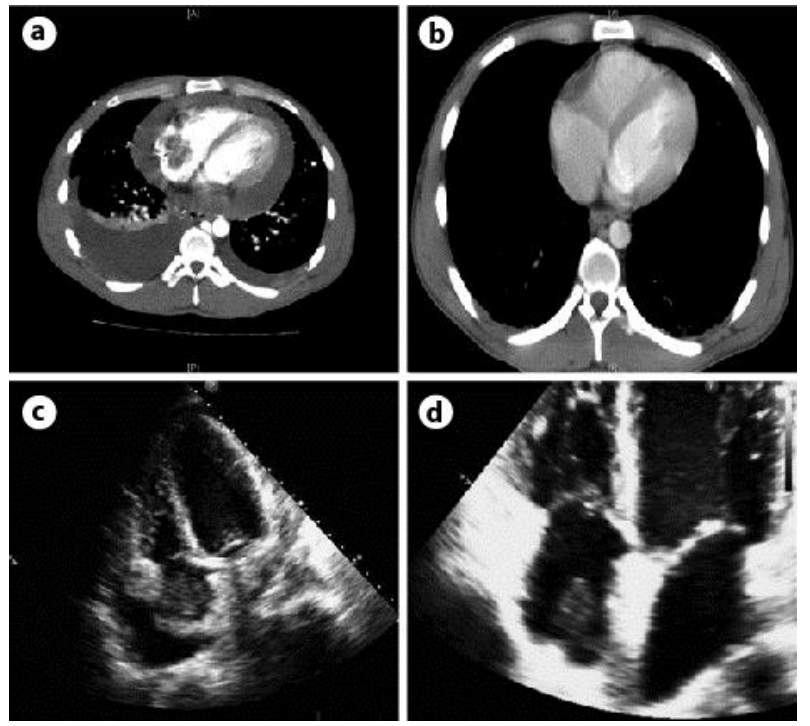

Fig. 1. a A chest CT scan with contrast showing a right atrial mass, a large pericardial effusion and bilateral pleural effusions. b A chest CT scan showing complete resolution of the bilateral pleural effusions 3 weeks after the first dose of combination chemotherapy. c An echocardiogram showing a large pericardial effusion with a large right atrial mass. $\mathbf{d}$ An echocardiogram showing complete resolution of the pericardial effusion and a significant decrease in the size of the right atrial mass 3 weeks after the first cycle of combination chemotherapy.

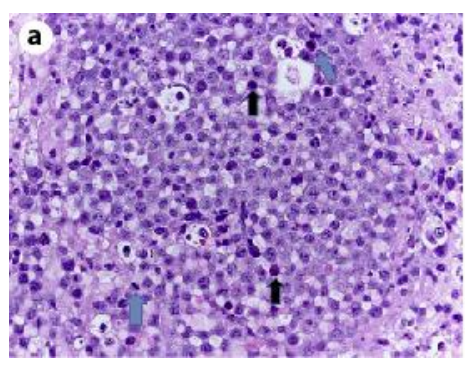

\section{b}
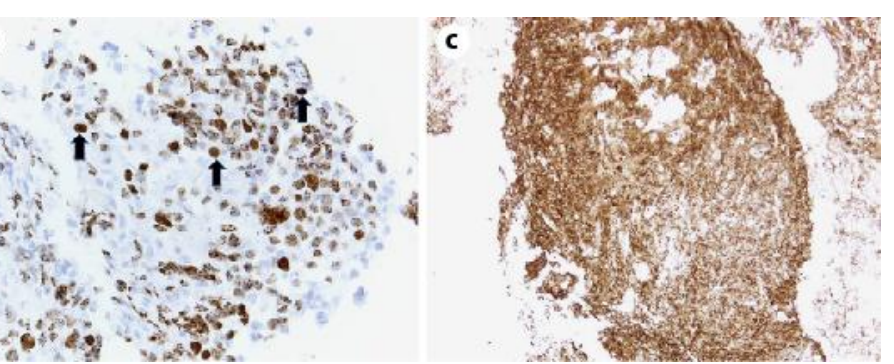

Fig. 2. a The hematoxylin and eosin-stained section shows a relatively monomorphous sheet of mediumsized atypical lymphocytes with round nuclei, fine to slightly vesicular chromatin, one or several prominent nucleoli and a moderate amount of slightly vacuolated pink cytoplasm. Mitotic activity is conspicuous (blue arrow). There are scattered necrotic and apoptotic cells (black arrow). b Positive nuclear staining for HHV-8 by immunohistochemistry (some examples of positive nuclei are marked by black arrows). c Cytoplasmic staining for the B cell marker CD20. 